\title{
The New 30 Doradus
}

\author{
Nolan R. Walborn and Rodolfo H. Barbá
}

STScI, 3700 San Martin Dr., Baltimore, MD 21218, USA

\begin{abstract}
Recent optical and infrared studies of 30 Doradus have revealed a more complex and diverse star-formation history than previously recognized. Five spatially and/or temporally distinct populations have been identified, including a new generation currently being triggered by the R136 cluster in its surroundings. HST/NICMOS observations of the latter provide insights into the phenomena of triggered massive-star formation, as well as the global structure and evolution of the starburst region.
\end{abstract}

\section{Stellar Populations}

A groundbased, blue-violet spectral classification study of the 30 Doradus stellar content by Walborn \& Blades (1997) has revealed five spatially and/or temporally distinct components:

1. The central ionizing cluster including R136 corresponds to the Carina phase of OB cluster evolution, with an age of 2-3 Myr. HST/FOS spectroscopy by Massey \& Hunter (1998) has shown that two-thirds of the 60 brightest stars in and around R136 are of spectral type O3, by far the richest concentration known of these hottest and most massive young stars.

2. A younger, Orion-phase generation of age $<1 \mathrm{Myr}$, in or near the bright nebular filaments west and northeast of R136, containing heavily embedded early-O dwarfs and IR sources, has likely been triggered by the central cluster. This component is further discussed in the second section below.

3. An older population of late-O and early-B supergiants throughout the central field represents the Scorpius OB1 phase with age 4-6 Myr and has no clear structural relationship to the younger groups. These stars may simply belong to the larger young region in which 30 Doradus is embedded.

4. The previously known, older still compact cluster Hodge $301,3^{\prime}$ northwest of R136, contains A and M supergiants and corresponds to the $h$ and $\chi$ Persei phase, of age $10 \mathrm{Myr}$.

5. A newly recognized Sco OB1-phase association surrounds the recently discovered Luminous Blue Variable R143 in the southern part of the Nebula.

Evidently, star formation has occurred in discrete events at different epochs in 30 Doradus. Global CMDs and IMFs of the region will be heterogeneous 
composites (see also Barbá \& Walborn in these proceedings), and there are clear implications for the interpretation of more distant starbursts. This entire region would subtend 0.5 at the Virgo Cluster.

\section{HST/NICMOS Views of the New Generation}

Recent optical, IR, and radio evidence for current star formation in 30 Doradus is reviewed by Rubio et al. (1998) and Walborn et al. (1999). The latter paper presents first results from an HST/NICMOS survey, which because of its 0.1 resolution provides new insights; some further observational details are given by Barbá et al. in these proceedings. Many new IR sources, including multiple systems, clusters, and nebular structures, are found in these NICMOS images. Knots 1-3 of Walborn \& Blades (1997), early O stars embedded in dense nebular knots, are all found to be compact multiple systems.

The first of the accompanying figures shows the field of Knots 1 and 2 (northeast of R136) in a WFPC2 $U, V, I$ composite, and the second in a NICMOS $J, H, K$ composite with the same orientation and scale; the scale bars indicate $N, E$ and are $4^{\prime \prime}=1 \mathrm{pc}$ in length. Knot 1 resides at the top of a massive dust pillar oriented directly toward R136, whose summit has just been removed, exposing the newborn stellar system. Two of the brightest IR sources in 30 Dor (arrowed in the NICMOS figure), with only very faint counterparts in $I$, are found in an adjacent pillar with the same orientation just northeast of Knot 1 . Another very bright IR source is within the head of a pillar near Knot 3 also oriented toward R136 (west of R136, not shown here), while parsec-scale jet structures have been discovered in association with Knots 2 and 3 . The Knot 2 structures, arrowed in the accompanying NICMOS figure, consist of detached, nonstellar IR sources aligned on either side of the stellar system, which are interpreted as impact points of a highly collimated, possibly rotating bipolar jet on the surrounding dark clouds; there is also an $\mathrm{H}_{2} \mathrm{O}$ maser in this field.

The outflows from young massive stars in 30 Dor are the first extragalactic examples of the phenomenon. These results establish the 30 Doradus Nebula as a prime region in which to investigate the formation and very early evolution of massive stars and multiple systems; several sequential stages and their interstellar interactions are directly observable in the optical and IR. The theme of triggered formation within the heads of extensive dust pillars oriented toward R136 is strong. In addition, these results provide further insights into the global structure and evolution of 30 Doradus, which are significant in view of its status as the best resolved extragalactic starburst. As discussed by Scowen et al. (1998), the most intense nebular emission arises from filamentary interfaces between the central cavity and surrounding molecular clouds. The IR results show that these interfaces are in fact star-formation fronts moving into the dark clouds. 30 Doradus is a two-stage starburst, in which a second generation of massive stars is being triggered around the periphery of the initial, massive central cluster. It will evolve into a giant shell $\mathrm{H}$ II region like $\mathrm{N} 11$ in the LMC (Walborn \& Parker 1992) and NGC 604 in M33.

Acknowledgments. Support for this work was provided by NASA through grants numbers GO-7819.01-96A and AR-7545.01-96A from STScI, which is operated by AURA, Inc., under NASA contract NAS 5-26555. 


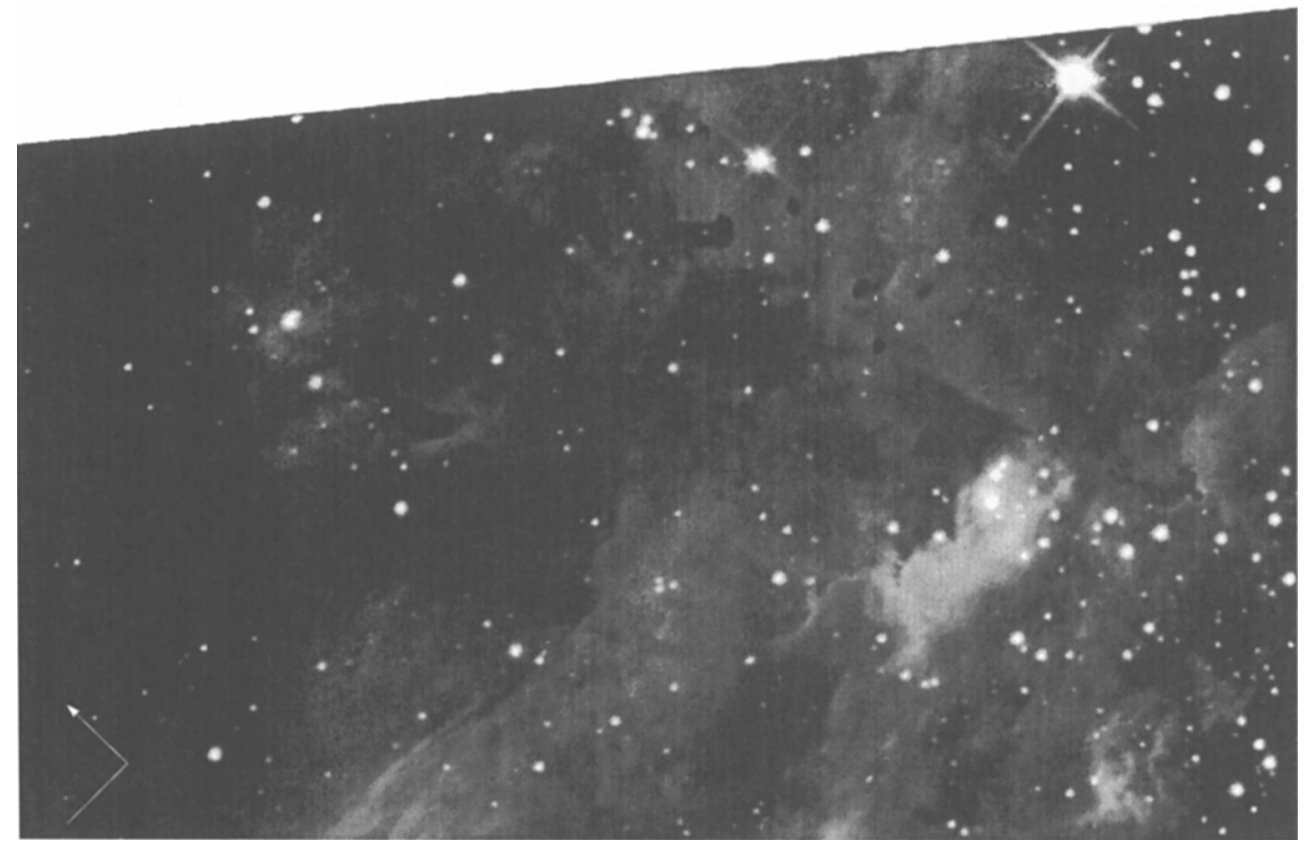

Figure 1. The field of Knots 1 and 2 northeast of R136 in a WFPC2 $U, V, I$ composite image.

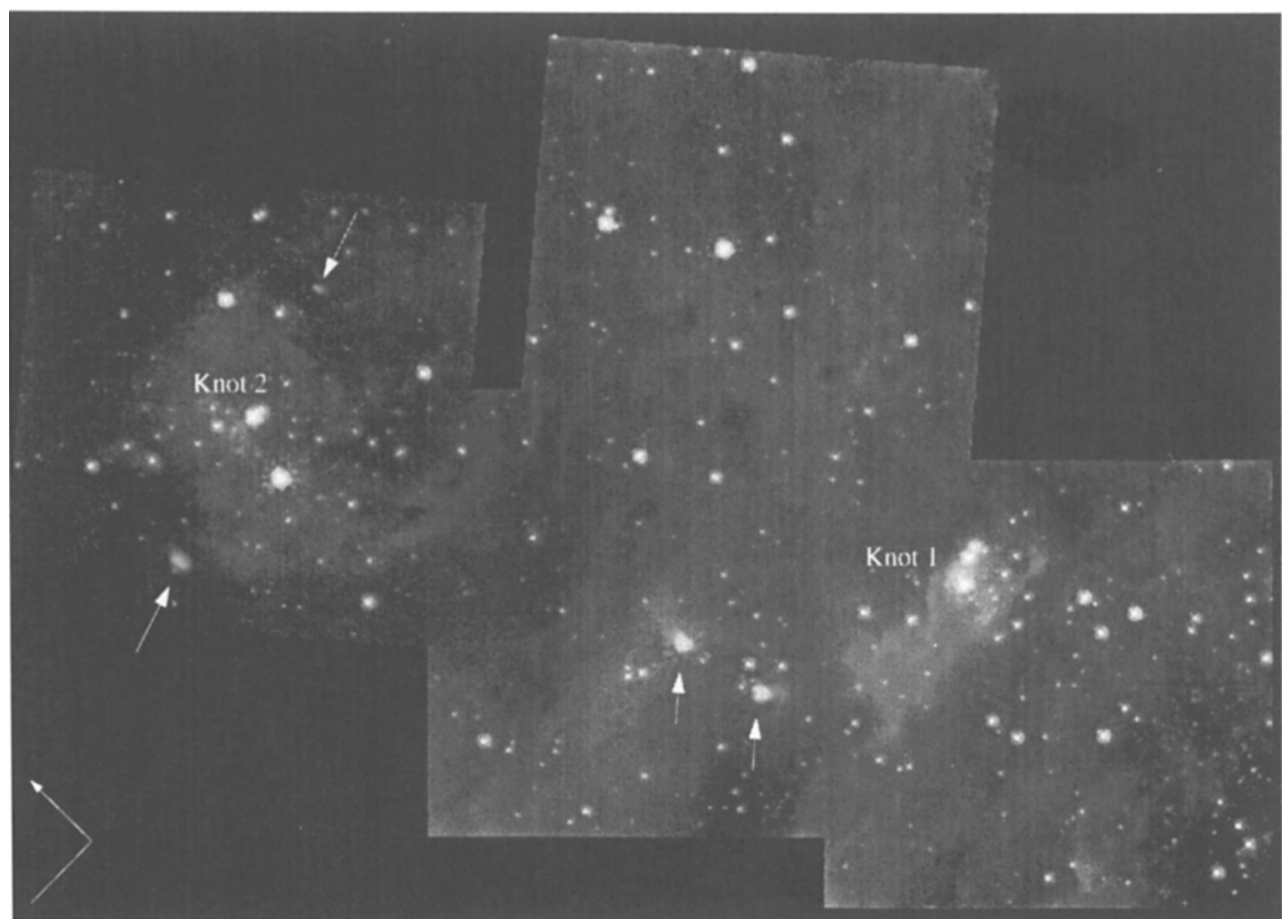

Figure 2. As in Fig 1., but in a NICMOS $J, H, K$ composite image. 


\section{References}

Rubio, M., Barbá, R.H., Walborn, N.R., Probst, R.G., García, J., \& Roth, M.R. 1998, AJ, 116, 1708

Massey, P., \& Hunter, D.A. 1998, ApJ, 493, 180

Scowen, P.A., et al. 1998, AJ, 116, 163

Walborn, N.R., Barbá, R.H., Brandner, W., Rubio, M., Grebel E.K., \& Probst, R.G. 1999, AJ, 117, 225

Walborn, N.R., \& Blades, J.C. 1997, ApJS, 112, 457

Walborn, N.R., \& Parker, J.Wm. 1992, ApJ, 399, L87 